\title{
Research Note: Bird-Resistant Pollination Bags for Sorghum Breeding and Germplasm Maintenance
}

\author{
Dennis C. Gitz III ${ }^{1 *}$, Jeffrey T. Baker ${ }^{2}$, Zhanguo Xin ${ }^{1}$, Robert J. Lascano ${ }^{1}$, John J. Burke ${ }^{1}$, \\ Sara E. Duke ${ }^{3}$ \\ ${ }^{1}$ USDA-ARS ${ }^{\#}$, Cropping Systems Research Laboratory, Lubbock, USA; ${ }^{2}$ USDA-ARS, Cropping Systems Research Laboratory, Big \\ Spring, USA; ${ }^{3}$ USDA-ARS, Southern Plains Area, College Station, USA. \\ Email: ${ }^{*}$ dennis.gitz@ars.usda.gov
}

Received January $4^{\text {th }}, 2013$; revised February $10^{\text {th }}, 2013$; accepted February $27^{\text {th }}, 2013$

\begin{abstract}
Bird damage is a problem in sorghum breeding and germplasm maintenance operations. Paper pollination bags are damaged by rain and provide only a minimal deterrent to birds. To overcome these limitations we fabricated pollination bags from spun polyethylene fiber sheeting. No seed yield difference was found between plants bagged with either spun polyethylene or paper. Seed loss by bird damage was nearly eliminated under the polyethylene bags. In areas where bird damage is problematic bird resistant pollination bags can allow for a reduction in the plot size required for breeding and germplasm maintenance operations, increase the productivity of such operations as genetic diversity per unit land area, and make direct measurement of seed yield possible in agronomic field experiments.
\end{abstract}

Keywords: Sorghum; Breeding; Pollination Bags; Bird Damage

\section{Introduction}

Sorghum (Sorghum bicolor L.) races exhibit considerable genetic and morphological diversity. Because of this diversity and because of the large number of mutants that have been developed with stable heritable traits, sorghum is a crop with considerable potential for improvement. Maintaining sorghum germplasm collections, characterizing and selecting mutants, and carrying out classical breeding operations, requires systems that maximize the genotypic diversity in the field. This is usually accomplished by simply reducing plot size so that a large number of isolines or races occupy a given land area. In addition to allowing more genotypes to be maintained or increased, this reduces the inputs associated with land area and reduces manual labor associated with culturing sim-

\footnotetext{
*Corresponding Author.

"The US Department of Agriculture (USDA) prohibits discrimination in all its programs and activities on the basis of race, color, national origin, age disability, and where applicable, sex, marital status, familial status, parental status, religion, sexual orientation, genetic information, political beliefs, reprisal, or because all or part of an individual's income is derived from any public assistance program. (Not all prohibited bases apply to all programs.) Persons with disabilities who required alternative means for communication of program information (Braille, large print, audiotape, etc.) should contact USDA's TARGET Center at (202) 720-2600 (voice and TDD). To file a complaint of discrimination, write to USDA, Office of Civil Rights, 1400 Independence Avenue, S.W., Washington DC 20250-9410, or call (800) 795-3272 (voice) or (202) $720-6382$ (TDD). USDA is an equal opportunity provider and employer.
}

ply by bringing plots closer to one another. Typically, sorghum of a given genotype is grown in a plot consisting of short single row adjacent to rows of different genotypes. Since outcrossing can be as high as $26 \%$ in such configurations [1], covering the panicles with paper "pollination bags" has been practiced for nearly a century where self pollination or "selfing" is required [2].

Bird damage to developing sorghum seeds is a problem worldwide [3]. In sorghum nurseries where small plots of tens of plants of a given genotype are grown, consumption of even a few plants' seed production by birds represents a considerable loss. Bird damage is a continuing problem in sorghum nursery operations conducted at the USDA-ARS location in Lubbock, TX.

The Lubbock facility embedded within a suburban area at the edge of urban center of about 100 square kilometers (Lat. 33.594657 ${ }^{\circ}$, Long. $-101.900232^{\circ}$ ). The nursery is proximal to several water sources of water and represents an isolated patch of particularly plentiful food resources for migrating native and invasive dove as well as other species such as grackles, blackbirds and sparrows. Attempts to protect developing seeds from damage have traditionally been by covering the developing panicles with heavy weather resistant paper pollination bags. Such attempts have been of limited effectiveness. Birds would simply tear the paper to access the developing grain underneath. It appeared that the birds subsequently became conditioned and associated paper pollination 
bags with food. If a wooden stake or denuded sorghum stalk was topped with a pollination bag the birds would tear it as well. Other attempts to limit bird damage included placing inflatable snakes, highly reflective sheets of foil or aluminized plastic that move in the wind, sound generators designed to randomly startle the birds, and spraying the bags with commercial chemical "bird repellents". These proved ineffective as well. To control dove, growers in the region typically simply open the land to upland bird hunters, which limits bird damage and also provides an additional source of revenue. Because our facility lies within the city limits the discharging firearms is prohibited by local law. Moreover the policies at federal research institutions and most universities surrounding the use or even simple possession of firearms limit or prohibit such approaches. Researchers eventually resorted to simply walking through the entire field daily and, when pollination bags were found to be damaged or destroyed (Figure 1(A)), workers simply placed another paper bag over the damaged bag or panicle. Such an approach does not prevent, but controls damage after it has occurred and so it also makes direct measurements of seed yield problematic if not impossible.

As part of a larger project we needed to increase selected sorghum seed lines to tens of kilograms to allow the use of a conventional seed drill the following year. Because we had experienced considerable bird damage in the past and wanted to maximize yield from the limited plot space available we wished to purchase inexpensive bird resistant pollination bags. After searching for an off the shelf commercial solution without success we approached several bag manufacturers inquiring about custom production of pollination bags to our specifications. Extremely limited interest was expressed in developing bags for the niche market of sorghum breeding. For these reasons it was decided to simply fabricate bird resistant pollination bags in the lab.

\section{Methods}

\subsection{Pollination Bag Fabrication}

Pollination bags were fabricated from spunbond polyethylene fiber sheet designed and marketed as a vapor barrier for residential building construction (Tyvek ${ }^{1}$ Homewrap, DuPont Corp., Wilmington DE). A roll of the material was purchased at the local "cash and carry" building supply store and was cut into $32 \mathrm{~cm}(13 ")$ widths by placing and securing hose clamps at the desired spacing along the roll to serve as a guide and to prevent unwinding. The roll was cut with a fine toothed hacksaw blade lubricated with paraffin that was frequently reapplied

\footnotetext{
${ }^{\mathrm{T}}$ Mention of trade names or commercial products in this publication is solely for the purpose of providing specific information and does not imply recommendation or endorsement by the US Department of Agriculture.
}

during cutting. The resulting $32 \mathrm{~cm}$ rolls were each cut into $38 \mathrm{~cm}\left(15^{\prime \prime}\right)$ pieces, using a jig that supported the roll on a dowel and which allowed the material to be fed under a piece of aluminum bar stock. In operation, the material was simply pulled to a mark on the plywood base and cut along the bar- stock straight edge and the action repeated. The resulting pieces were folded lengthwise keeping proprietary product identification visible and sewn about $0.5-1.0 \mathrm{~cm}$ from the edge leaving one short edge open. The resulting $15 \mathrm{~cm} \times 37 \mathrm{~cm}$ bags were turned inside-out so that product identification and trademarking were on the interior of the bag and a pristine bright white surface was left on the outside of each bag. This was done to minimize absorbed radiation and to accept and maximize visibility of hand labeling done with felt tip indelible markers. While this material allows for the passage of water vapor we lightly perforated each bag with a single pass of a handheld pin-pricker designed for perforating wall paper.

\subsection{Plant Culture}

Sorghum (cvar BT $\times 623$ ) was field grown in five $5 \times 10$ $\mathrm{m}$ plots. After emergence the plants were thinned so that the remaining plants were distributed along a $32 \mathrm{~cm} \times 32$ $\mathrm{cm}$ rectilinear array. Shortly after boot, spun polyethylene or paper bags were placed on the developing panicles, the bag opening folded about the stem and the opening stapled so the bag would not come off in high winds. When the spun polyethylene bag supply was exhausted paper bags continued to be used. As bird damage occurred to the paper bags, additional paper bags were

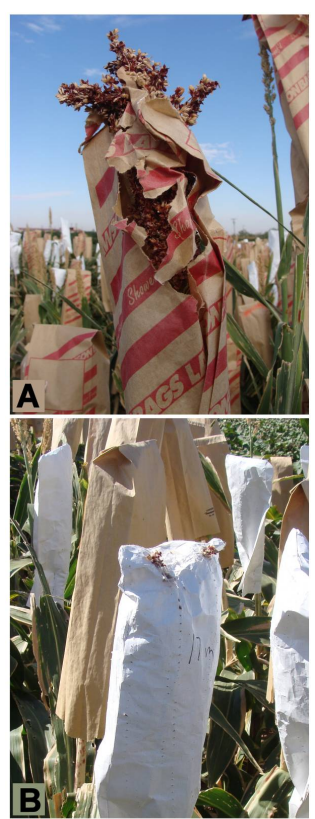

Figure 1. Bird damage to (A) paper and (B) spun polyethylene pollination bags. 
placed over the damaged bags without stapling.

\subsection{Seed Yield and Development}

At harvest, panicles under twenty spun polyethylene or twenty paper bags undamaged by birds, were collected from the interior of each plot, and individually mechanically threshed. Undamaged paper bags were easily identified as a panicle covered by single stapled bag, while damaged bags were covered by bags without staples. During threshing, seeds from each panicle were placed in envelopes and removed to the lab where the seeds were stored until they could be weighed and counted.

The number of paper bagged plants ranged from 80 to 170 plants/plot and averaged about 100/plot. The bags were scored as undamaged (the original bags intact), slightly damaged (original bags having holes no larger than a workers thumb would fit), or severely damaged (those having considerably larger, more numerous holes, or shredded). The sorted bagged heads were counted and the percentage of exhibiting each damage level was calculated. The spun polyethylene bags were not scored because damage exhibited was negligible.

At the conclusion of the field season the bags were inverted (turned inside-out), placed in an automatic clothes washing machine, washed in a solution of clothing detergent and sodium hypochlorite bleach, allowed to air dry on the bench, and stored for next season's use.

\subsection{Statistical Analysis}

Results for each bag type were simply averaged for each plot and characterized by calculating averages and standard error across plots or, where differences were examined, by Student's t-test ( $\mathrm{n}=5$ plots $)$.

\section{Results and Discussion}

Birds accessed sorghum under about half of the paper pollination bags (Table 1). Very few bags were only slightly damaged, perhaps when birds continued feeding and were infrequently startled by workers or equipment. Most bags that were damaged were damaged extensively before being subsequently covered with a fresh bag. This suggested that once birds started feeding on sorghum under a pollination bag they persisted feeding and either shredded the bags or accessed the bags though multiple holes. The birds then continued to feed until the panicle seed load had been considerably reduced, the site didn't provide sufficient reward for the effort expended, or for some other reason, perhaps when they were startled by field personnel. Different bird species exhibited different feeding behaviors. The mexican grackles (Quiscalus mexicanus) were especially destructive and would open the tops of paper pollination bags and subsequently either peel the bags away from the panicle, or even open the top and pull the resulting open sleeve down the stem exposing the entire panicle. Damage to the polyethylene bags was not quantified, but of the hundreds used in the field perhaps six exhibited damage and this limited to a single to a few holes less than $0.5 \mathrm{~cm}$ in diameter (Figure 1(B)). This damage was attributed to the Mexican grackles. On these few instances the bird would tear at one of the pinholes we had pricked into the bags to facilitate gas exchange and open it just enough to insert its beak. In one case the thread stitching the bag seam was targeted by a bird and failed. Loss under was not quantified but was certainly negligible especially when compared to the paper bags.

No statistically significant effect $\left(\mathrm{P}_{\mathrm{t}}<0.05\right)$ on seed development or yield was observed (Table 2). Average seed size was reduced by about $5 \%$ by the spun polyethylene bags but this was of only modest significance $\left(\mathrm{P}_{t}=\right.$ 0.1 ) and was associated with a similar increase in mean seed numbers produced/plant of about $4 \%$ as compared to the paper bags.

The only other report of workers fabricating pollination bags from this material was for Hazelnut [4] where it was important that the bags remain rigid to prevent damaging the stigmas. For hazelnut the bags could be used only a single season or two and wind was a problem as it softened the bags. In the present study the bags were washed after use and afterwards were quite pliable and not nearly as "crisp"; they felt "softer" to the touch. Nevertheless, the bags were re-used in the field by other sorghum workers in our unit the following season and few problems were reported; though a systematic study has not been undertaken comparing washed vs. pristine unwashed bags.

Table 1. Bird damage to paper pollination bags. Mean values and standard error are shown $(n=5$ plots).

\begin{tabular}{ccc}
\hline Damage Level $^{\mathrm{a}}$ & \% of Bags & S.E. \\
\hline None & 40.8 & 8.4 \\
Moderate & 10.2 & 1.4 \\
Severe & 49.0 & 8.9 \\
\hline
\end{tabular}

${ }^{\mathrm{a}}$ None $=$ No damage Moderate $=$ One or two hole(s) no larger than diameter of a thumb; Severe = Many Holes or bag shredded.

Table 2. Effects of pollination bag material on seed yield and development. Mean values and standard error are shown ( $=5$ plots).

\begin{tabular}{cccc}
\hline Parameter & Paper & Tyvek & $\mathbf{P}_{\mathbf{t}}$ \\
\hline Seed Size $\left(\mathrm{mm}^{3}\right)$ & 38.42 & 35.87 & 0.10 \\
Yield $(\mathrm{g} / \mathrm{plant})$ & 69.21 & 65.49 & 0.063 \\
Yield $\left(\mathrm{cm}^{3} /\right.$ plant $)$ & 88.58 & 85.57 & 0.76 \\
Seeds/Plant & 2527.46 & 2635.89 & 0.75 \\
\hline
\end{tabular}




\section{Conclusion}

These bags are easily and quickly made and could serve as a suitable alternative to paper pollination bags. The materials alone cost about $\$ 0.20 / \mathrm{bag}$ which is a bit more than three times the cost of a heavy paper pollination bag, not including labor. However, they practically eliminate loss to bird damage so the cost of additional bags is saved. Perhaps most important, using bird-proof bags eliminates the need for laborers to repeatedly walk the field to cover plants with additional bags as bird damage occurs during seed development. If it is critical to protect germplasm from birds when multiplying germplasm in field settings or to obtain reliable estimates of yield in agronomically relevant conditions, these bags provide a simple effective solution.

\section{Acknowledgements}

We thank Dr. Lan Liu-Gitz for helpful discussions and suggestions through the course of this experiment and
Cody Byars for his technical assistance. This work was funded in part by the USDA-ARS Ogallala Aquifer Program.

\section{REFERENCES}

[1] J. F. Pedersen, J. J. Toy and B. Johnson, "Natural Outcrossing of Sorghum and Sudangrass in the Central Great Plains," Crop Science, Vol. 38, No. 4, 1998, pp. 937-939. doi:10.2135/cropsci1998.0011183X003800040009x

[2] A. H. Leidigh, "Methods for the Improvement of Sorghum," Journal of Heredity, Vol. 2, No. 4, 1911, pp. 294 296.

[3] S. J. Ormerod and A. R. Watkinson, "Editor's Introduction: Birds and Agriculture," Journal of Applied Ecology, Vol. 37, No. 5, 2000, pp. 699-705. doi:10.1046/j.1365-2664.2000.00576.x

[4] D. C., Smith and S. A. Mehlenbacher, "Use of Tyvek Housewrap for Pollination Bags in Breeding Hazelnut (Corylus avellana L.)," Hortscience, Vol. 29, No. 8, 1994, p. 918 . 